\title{
Colorectal Transit and Volume During Treatment With Prolonged-release Oxycodone/Naloxone Versus Oxycodone Plus Macrogol 3350
}

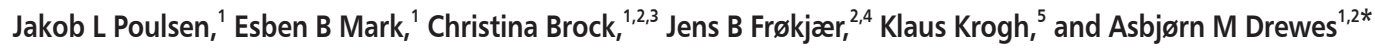 \\ ${ }^{1}$ Mech-Sense, Department of Gastroenterology and Hepatology, Aalborg University Hospital, Aalborg, Denmark; ${ }^{2}$ Department of Clinical \\ Medicine, Aalborg University, Aalborg, Denmark; ${ }^{3}$ Department of Drug Design and Pharmacology, University of Copenhagen, Copenhagen, \\ Denmark; ${ }^{4}$ Mech-Sense, Department of Radiology, Aalborg University Hospital, Aarhus, Denmark; and ${ }^{5}$ Neurogastroenterology Unit, Department \\ of Hepatology and Gastroenterology, Aarhus University Hospital, Aarhus, Denmark
}

\section{Background/Aims}

Opioid-induced constipation (OIC) is the most common gastrointestinal (GI) side effect to opioid treatment. Opioid receptor antagonists against OIC have been introduced, but their efficacy has not been directly compared to conventional laxatives. Our aim was to compare symptoms and objective parameters of gut function in an experimental model of OIC during treatment with the opioid antagonist naloxone and oxycodone in prolonged-release (PR) formulation versus oxycodone plus macrogol 3350.

\section{Methods}

In this randomized, double-blind, crossover trial 20 healthy men received a 5-day treatment of combined PR oxycodone/naloxone or PR oxycodone plus macrogol 3350. Regional GI transit times and segmental colorectal transit were assessed with the Motilis 3D-Transit electromagnetic capsule system. Colorectal volumes were determined by MRI. OIC symptoms were assessed with validated questionnaires, along with stool frequency and consistency.

\section{Results}

Total colorectal volume did not change after 5 days' treatment with PR oxycodone/naloxone (941 vs $1036 \mathrm{~mL} ; P=0.091$ ), but increased significantly after PR oxycodone plus macrogol treatment (912 vs $1123 \mathrm{~mL} ; P<0.001)$. Neither regional Gl transit times nor segmental colorectal transit differed between the treatments (all $P>0.05$ ). The Patient Assessment of Constipation Symptom Questionnaire abdominal symptoms score was lower during PR oxycodone/naloxone compared to PR oxycodone plus macrogol (0.2 vs $3.2 ; P=0.002)$. Stool frequency was lower during PR oxycodone/naloxone compared to PR oxycodone plus macrogol (4.2 vs $5.4 ; P=$ $0.035)$.

\section{Conclusions}

PR oxycodone plus macrogol increases colorectal volume, but does not improve Gl transit compared to PR oxycodone/naloxone. However, PR oxycodone/naloxone results in a lower abdominal symptom burden, despite higher stool frequency during macrogol treatment.

(J Neurogastroenterol Motil 2018;24:119-127)

\section{Key Words}

Constipation; Motility; Naloxone; Opioids; Oxycodone

Received: April 24, 2017 Revised: August 21, 2017 Accepted: October 13, 2017

(.) This is an Open Access article distributed under the terms of the Creative Commons Attribution Non-Commercial License (http://creativecommons. org/licenses/by-nc/4.0) which permits unrestricted non-commercial use, distribution, and reproduction in any medium, provided the original work is properly cited.

*Correspondence: Asbjørn M Drewes, MD, PhD, DMSc

Mech-Sense, Department of Gastroenterology and Hepatology, Aalborg University Hospital, Mølleparkvej 4, DK-9000 Aalborg, Denmark Tel: +45-9766-3562, Fax: +45-9766-3577, E-mail: amd@rn.dk 


\section{Introduction}

Treatment with opioids is essential in patients with severe acute and chronic pain. However, opioid treatment often results in substantial gastrointestinal (GI) side effects, collectively referred to as opioid-induced bowel dysfunction (OIBD). ${ }^{1}$ These effects are mediated by binding of opioids to peripheral $\mu$-opioid receptors in the gut, thereby disturbing normal GI motility and function. ${ }^{2}$ The result is symptoms such as dry mouth, gastroesophageal reflux, vomiting, bloating, abdominal pain, anorexia, hard stools, and incomplete evacuation. The most common symptom is however opioid-induced constipation (OIC), occurring in $15 \%$ to $90 \%$ of the patients. ${ }^{3}$

Recommended first-line treatment for OIC is conventional laxatives. ${ }^{4}$ However, there are no randomized controlled, doubleblind trials to support their efficacy in OIC. The pathophysiology behind OIC differs from that of functional constipation and OIC may not respond adequately to conventional laxatives. ${ }^{5}$ Hence, many patients with OIC have GI symptoms despite the use of 2 or more laxatives. ${ }^{5,6}$ Furthermore, laxatives often cause side effects, such as bloating, abdominal distension, rumbling, flatulence, and gastroesophageal reflux. ${ }^{7}$

Several opioid receptor antagonists directly targeting the pathophysiology behind OIC have been introduced. Initial results have been promising, ${ }^{8,9}$ but head-to-head comparison of these opioid antagonists versus conventional laxatives has not been conducted. Furthermore, considerable diversity in outcome measures used in previous trials makes comparison of efficacy difficult.

The recently suggested definition for OIC by the Rome IV working group is an important step towards easier comparison of trials evaluating OIC treatment, ${ }^{10}$ but these should ideally also include objective assessments. We have previously developed a model of OIC in healthy volunteers and shown that oxycodone causes symptoms of OIC, prolongs regional GI transit times, ${ }^{11}$ and increases colorectal volumes. ${ }^{12}$ The model for OIC in healthy volunteers allows for interventional studies of OIC not influenced by confounders such as comorbidity, activity level or concomitant drug that can all cause constipation and hence make studies in patients difficult to interpret.

We hypothesized that combined prolonged-release (PR) oxycodone/naloxone tablets would be more effective than PR oxycodone plus conventional laxative treatment with macrogol 3350 in preventing oxycodone-induced OIC. To test this hypothesis, the aims of the study were to evaluate how the 2 treatments affected (1) regional GI transit times and segmental colorectal transit, (2) segmental colorec- tal volumes, (3) symptoms of OIC, and (4) associations between symptoms and the objective assessments.

\section{Materials and Methods}

\section{Study Design}

This randomized, double-blind, double-dummy, crossover trial was conducted in compliance with the principles of the International Conference on Harmonisation on Good Clinical Practice of the European Union, the Danish Health and Medicines Authority and approved by the local Ethical Committee (N-20130030). Participants were enrolled from September 2015 to June 2016 at MechSense, Department of Gastroenterology and Hepatology, Aalborg University Hospital. Data reported in the present paper are part of a larger protocol that simultaneously evaluated treatment of experimentally induced OIBD on a number of parameters (unpublished data). The full trial is registered at www.clinicaltrialsregister.eu (EudraCT No. 2013-001540-60).

Participants were all healthy volunteers treated for 5 days (one period) with (1) combined PR oxycodone/naloxone tablets or (2) PR oxycodone tablets and co-administered macrogol (polyethylene glycol) 3350 powder for oral solution. There was a wash-out period of at least 9 days, before the participants were crossed over to the other treatment. Participants were randomized by means of a computer-generated block-randomization list. The personnel who generated the randomization list were not otherwise involved in the study. Assignment to each randomization number was done chronologically in the same order participants were enrolled. Dropouts were replaced using mirror-randomization. The sample size calculation was based on a previous study using the same endpoints. ${ }^{13}$ Performing a sample size calculation with a mean difference of 0.5 days in total transit time between treatments, a variance of 0.4 , a power of $95 \%$ and $\alpha$ at 0.05 in a two-sided test, the calculation estimated a sample of 19 as appropriate. Thus, we choose a sample size of 20 participants.

Both treatment periods commenced with fasting baseline assessments of segmental colorectal volumes with MRI and questionnaires (Fig. 1). The first dose of the drugs was administered orally after baseline measurements (day 1, Mondays) and then repeated every 12th hour until the morning of day 5 (Fridays). Following administration of the first dose after baseline assessments, the participants were discharged from the research facility and returned to their daily activities. Three hours after administration of the first dose, a standardized meal $(375 \mathrm{kcal}, 11.4 \mathrm{~g}$ fat, $1.8 \mathrm{~g}$ fiber) was ingested and immediately after the meal was finished, 


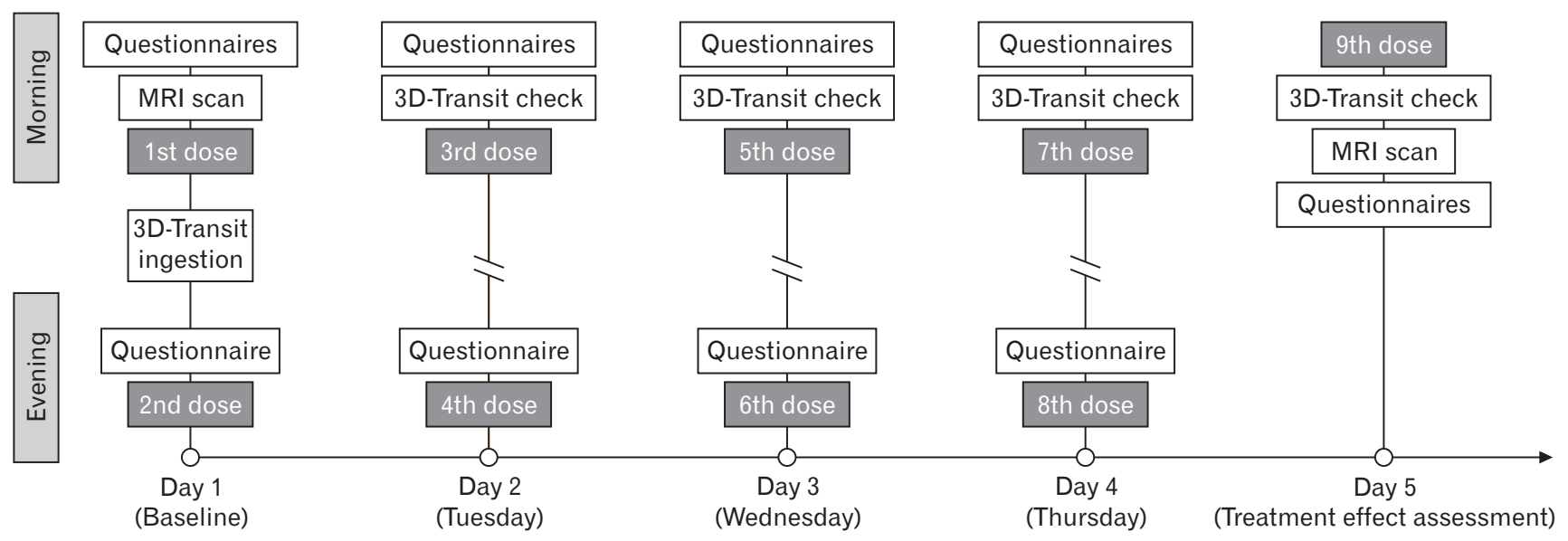

Figure 1. Timeline for one study period. MRI scans were done in fasting state at the same time at baseline and day 5. The 3D-Transit capsule was ingested 3 hours after administration of the first dose. Daily verification of the 3D-Transit capsule progression was done to confirm retention or expulsion from the body.

the electromagnetic 3D-Transit capsule (see below) was ingested with a glass of water. Participants returned to the research facilities on days 2, 3, and 4 for dosing, questionnaires, and so that emitted signals from the capsules could be monitored in real-time. This was done to confirm retention or expulsion of the capsule. Subjects were asked to refrain from hard physical work and sports. On day 5 , the measurements described for the baseline day were repeated. If the 3D-Transit capsule had not been expelled on day 5, the MRI was cancelled, as the capsule is not MRI compatible. Oxycodone dose in both periods was $5 \mathrm{mg}$ twice daily on day 1 and $10 \mathrm{mg}$ twice daily day 2 through day 4 and finalizing with $10 \mathrm{mg}$ on the morning of day 5 administered at least 1 hour before measurements. One sachet of macrogol 3350 powder for oral solution or matching placebo (dextrose) was co-administered with every dosing. Each sachet contained $13.125 \mathrm{~g}$ macrogol 3350, $350.7 \mathrm{mg}$ sodium chloride, $178.5 \mathrm{mg}$ sodium bicarbonate, and $46.6 \mathrm{mg}$ potassium chloride. To mask the different taste of macrogol or placebo each sachet was diluted in in $250 \mathrm{~mL}$ Rynkeby orange juice (Rynkeby, Denmark). ${ }^{14}$ PR oxycodone/naloxone tablets and matching placebo tablets were provided by Mundipharma Research Ltd, Cambridge, England. Macrogol and matching placebo were supplied by The Capital Region Pharmacy, Herlev, Denmark. For readability, the PR oxycodone/naloxone treatment period will from now on be referred to as "PR naloxone treatment" and the PR oxycodone plus macrogol 3350 treatment period as "macrogol treatment."

\section{Study Participants}

Eligible participants were healthy, male volunteers with neither current symptoms nor history of GI disease. Participants under- went a screening session where informed consent was obtained, and a physician evaluated their medical history to ensure that all inclusion and exclusion criteria were fulfilled. If found eligible, the participants were enrolled in the study.

Inclusion criteria were: (1) signed written informed consent declaration, (2) capable of reading and understanding Danish, (3) male of Northern European descent, (4) understanding of the study procedures, (5) age 20-60 years, (6) healthy, and (7) opioid naïve. The exclusion criteria were: (1) known allergy towards opioids, (2) participation in any other studies within 14 days of enrolment, (3) planned medical/surgical treatment within the study duration, (4) a need to operate heavy machinery or motor vehicles during the study, (5) any previous or current drug abuse, (6) non-removable piercings or metal implants, (7) daily alcohol or nicotine consumption, (8) any known disease that may influence the results, and (9) the use of prescribed medicine and/or herbal medicine.

\section{Study Outcomes}

\section{Gastrointestinal transit times}

Total and segmental GI transit times (gastric emptying, small intestinal transit, and colorectal transit) were determined using the 3D-Transit system. ${ }^{15}$ In short, the 3D-Transit system is a minimally invasive, ambulatory system, which relies on small, wireless telemetric capsules that are ingested and an extracorporeal, portable detector that tracks the precise position and general orientation of the capsule from ingestion to expulsion. ${ }^{16,17}$ Accordingly, changes in position, velocity of movements, and orientation of the capsules, reflecting gut contractile activity, and progression dynamics, may be 
studied with respect to anatomical information. ${ }^{16,18}$

Segmental transit times of the colon (cecum/ascending colon, transverse colon, descending colon, and rectosigmoid colon) were evaluated based on visualization and determination of the trajectory transversed in the colon by the 3D-Transit capsule, as described previously. ${ }^{11}$ If the capsule was not expelled on day 5, the recording was stopped, and the time of last confirmed signal from the capsule was used as a proxy for earliest possible expulsion time.

\section{Segimental colorectal volumes}

Segmental colorectal volumes were determined by MRI scans acquired using a 3T GE scanner (GE Signa HDxt, General Electric, Milwaukee, WI, USA). Approximately 40 contiguous coronal T2-weighted images with $4 \mathrm{~mm}$ slice thickness and in-plane resolution of approximately $0.9 \times 0.9 \mathrm{~mm}$ were obtained covering the entire colon and rectum. Segmental colorectal volumes (cecum/ ascending colon, transverse colon, descending colon, and rectosigmoid colon) were determined using semi-automatic in-house data analysis software, as described in detail previously. ${ }^{19,20}$

\section{Questionnaires}

The participants were asked to fill in 4 validated GI questionnaires: ${ }^{21}$ (1) the Bowel Function Index (BFI); a 3-item questionnaire specifically developed to assess OIC, (2) the Gastrointestinal Symptom Rating Scale (GSRS); covering 15 items assigned to 5 dimensions: gastroesophageal reflux, abdominal pain, dyspepsia, diarrhea, and constipation, (3) the Patient Assessment of Constipation Symptom Questionnaire (PAC-SYM); comprised of 12 items assigned to 3 subscales: stool symptoms, rectal symptoms, and abdominal symptoms, and (4) the Bristol Stool Form Scale evaluating stool frequency and consistency. The BFI and GSRS were administered at baseline and day 5, while the PAC-SYM and Bristol Scale were used to assess the dynamic changes of the general bowel function and thus assessed daily.

\section{Statistical Methods}

Data are presented as means (95\% confidence interval) unless otherwise indicated, and was assessed for normality and handled accordingly with parametric or nonparametric statistics.

Data from regional GI transit times and segmental colorectal transit were non-parametrically distributed, and are presented as medians and interquartile range. Wilcoxon signed rank test was used to compare the 2 treatments for non-parametric variables.

Segmental colorectal volumes, BFI, PAC-SYM, Bristol Scale scores, and GSRS were analyzed with repeated measures mixed models. PAC-SYM and segmental colorectal volume data were baseline corrected. PAC-SYM total score and the 3 subscale scores (abdominal symptoms, stool symptoms, and rectal symptoms) were analyzed in separate models. Data from the GSRS were analyzed as total score and syndrome dimension scores in separate models (abdominal pain, reflux, indigestion, diarrhea, and constipation). For mixed models with significant interaction, Bonferroni-adjusted post hoc tests were applied for multiple comparisons.

Pearson's product-moment correlation coefficient was used to identify linear associations between symptoms and objective assessments of constipation as determined by transit times and colorectal volumes.

All reported $P$-values were two-tailed and values less than 0.05 were considered significant. Data were analyzed using Stata version 14.2 (StataCorp LP, College Station, TX, USA).

\section{Results}

\section{Study Participants}

Twenty participants with a median age of 24 years (range: 18 to 40 years), a mean height of $182 \mathrm{~cm}(181-185 \mathrm{~cm})$, and mean body mass index of $23.8 \mathrm{~kg} / \mathrm{m}^{2}\left(22.6-25.0 \mathrm{~kg} / \mathrm{m}^{2}\right)$ were enrolled in the study. All participants completed both study periods. The 2 treatment periods were separated by a median of 45 days (range: $31-59$ days).

\section{Study Outcomes}

\section{Gastrointestinal transit times}

Forty capsules were ingested (1 per period for each subject) Due to technical problems in 1 case, it was only possible to determine total GI transit. Capsule retention on day 5 occurred in 9 recordings ( 5 during PR naloxone treatment and 4 during macrogol). In cases with capsule retention, the last confirmed signal from the capsule was used as proxy for the earliest possible expulsion time. Thus, it was possible to calculate the total transit in 40 recordings and regional GI transit in 39 recordings. No adverse events were registered.

There were no differences in total, regional GI, or segmental colorectal transit times between treatments (all $P>0.05$ ) (Table 1).

\section{Seǵmental colorectal volumes}

Five scans on day 5 in the PR naloxone period and 4 scans on day 5 in the macrogol period were not done due to retention of the 3D-Transit capsule. In addition, 3 scans were missed due to technical problems with the MRI scanner; one on day 5 in each of the 
treatment periods, and 1 at baseline in the macrogol period. Thus, 68 scans were available for further analysis with scans at all time points in 12 participants. Data form all 68 scans were included in the statistical model. There were no differences in total volume or in any of the segments between the 2 baselines (all $P>0.1$ ) (Table 2), or in the PR naloxone period (baseline vs day 5) (Table 2). In the macrogol period (baseline vs day 5), total colorectal volume and all segmental volumes increased significantly (Table 2). Baseline

Table 1. Total and Segmental Gastrointestinal Transit Times (in Hours) During the 2 Treatments

\begin{tabular}{lccc}
\hline & PR naloxone & Macrogol & $P$-value \\
\hline Gastric emptying time & $3.5(2.6-6.8)$ & $3.2(2.3-4.9)$ & 0.334 \\
Small intestine transit time & $5.3(4.7-6.3)$ & $5.8(4.6-8.1)$ & 0.091 \\
Cecum and ascending colon & $7.2(3.6-19.5)$ & $9.0(6.6-19.9)$ & 0.554 \\
Transverse colon & $4.5(1.6-9.5)$ & $6.8(2.8-9.1)$ & 0.331 \\
Descending colon & $1.9(0.1-7.7)$ & $4.9(1.1-10.4)$ & 0.917 \\
Rectosigmoid colon & $11.7(7.9-24.7)$ & $10.3(1.2-19.2)$ & 0.125 \\
Colorectal transit time & $39.6(26.6-74.3)$ & $38.2(30.9-61.2)$ & 0.872 \\
Total GI transit time & $46.0(34.8-84.7)$ & $46.6(41.6-70.7)$ & 0.793 \\
\hline
\end{tabular}

PR, prolonged-release; GI, gastrointestinal.

Data are presented as medians (interquartile range). corrected volumes increased significantly for macrogol compared to PR naloxone for the entire colorectum and for the rectosigmoid (Fig. 2). An example of a fully segmented colorectum is shown in Figure 3.

\section{Questionnaires}

No difference was found for BFI score between baselines $(P=$ $0.791)$ or between $P R$ naloxone and macrogol on day $5(P=0.666)$

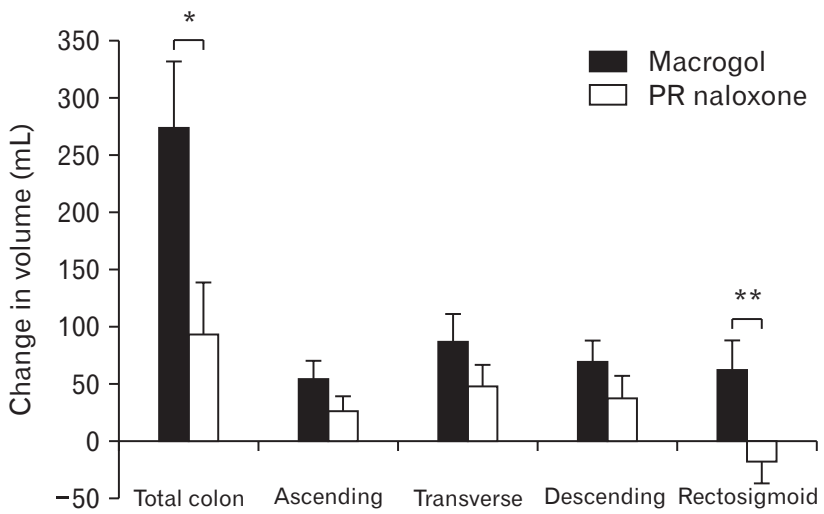

Figure 2. Change in total and segmental colorectal volumes during the 2 treatments. Data are presented as means \pm SEM. ${ }^{*} P=0.001$. ${ }^{*} P=0.005 . \mathrm{PR}$, prolonged-release.

Table 2. Segmental Colorectal Volumes (in $\mathrm{mL}$ ) for Baseline and Day 5 in Both Treatment Periods

\begin{tabular}{lccccc}
\hline & Cecum/ascending colon & Transverse colon & Descending colon & Rectosigmoid & Total \\
\hline PR naloxone (baseline) & $220 \pm 25$ & $258 \pm 42$ & $187 \pm 32$ & $276 \pm 60$ & $941 \pm 108$ \\
PR naloxone (day 5) & $257 \pm 41$ & $295 \pm 47$ & $210 \pm 51$ & $273 \pm 71$ & $1036 \pm 176$ \\
Change from baseline & $26 \pm 25(+17 \%)$ & $48 \pm 37(+14 \%)$ & $38 \pm 38(+12 \%)$ & $-18 \pm 36(-1 \%)$ & $94 \pm 88(+10 \%)$ \\
& $(P=0.156)$ & $(P=0.161)$ & $(P=0.384)$ & $(P=0.904)$ & $(P=0.087)$ \\
Macrogol (baseline) & $216 \pm 39$ & $270 \pm 59$ & $184 \pm 55$ & $242 \pm 55$ & $912 \pm 158$ \\
Macrogol (day 5) & $277 \pm 53$ & $328 \pm 51$ & $231 \pm 44$ & $287 \pm 52$ & $1123 \pm 145$ \\
Change from baseline & $54 \pm 31(+27 \%)$ & $87 \pm 47(+22 \%)$ & $70 \pm 35(+25 \%)$ & $63 \pm 49(+19 \%)$ & $274 \pm 113(+23 \%)$ \\
& $(P=0.005)$ & $(P=0.006)$ & $(P=0.022)$ & $(P=0.026)$ & $(P<0.001)$ \\
\hline
\end{tabular}

$\mathrm{PR}$, prolonged-release.

Data are presented as means $\pm 95 \%$ confidence interval (change from baseline in percent).

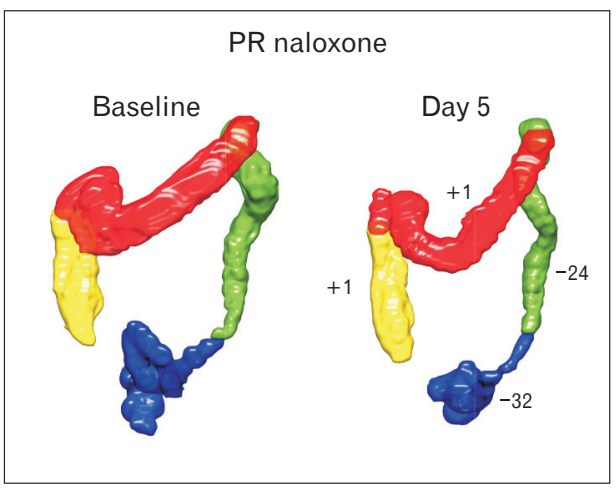

Figure 3. Fully segmented colorectal volumes for one representative participant on the 2 baseline days, and on day 5 during the 2 treatments. Numbers next to the colorectal segments on day 5 in each treatment period are change in volume (in $\mathrm{mL}$ ) from baseline. PR, prolonged-release. 
A

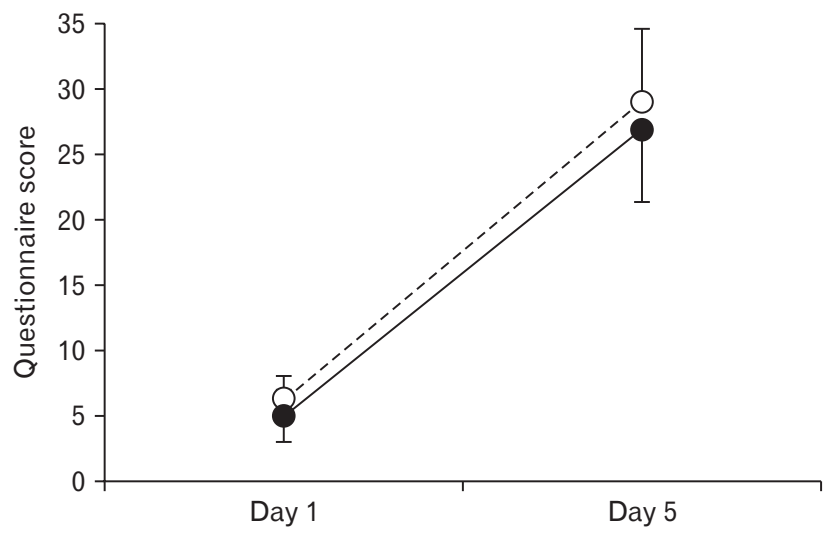

B

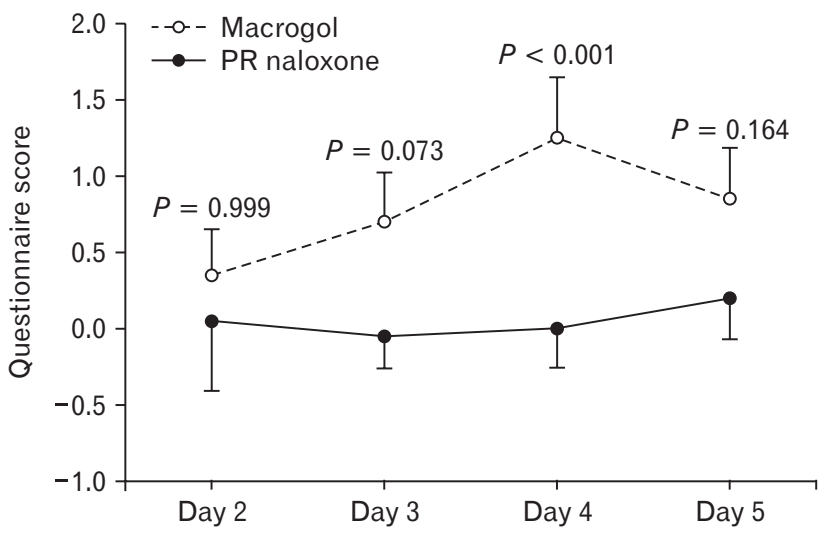

Figure 4. Development of gastrointestinal symptoms during the 2 treatments. Panel (A) shows development in Bowel Function Index (BFI) scores during the 2 treatments. There was no difference between the treatment periods. Panel (B) illustrates the baseline corrected Patient Assessment of Constipation (PAC-SYM) abdominal subscale scores during the treatments. The $P$-values are the Bonferroni-adjusted pairwise comparison of scores between days. Data are presented as means \pm SEM. PR, prolonged-release.

(Fig. 4A). Hence, there was no difference between the treatment periods. BFI score increased significantly from baseline to day 5 during both $\mathrm{PR}$ naloxone $(P<0.001)$ and macrogol $(P<0.001)$ (Fig. 4A).

For the baseline corrected PAC-SYM scores, there was no difference in total score during PR naloxone compared to macrogol $(8.5 \pm 6.9$ vs $4.8 \pm 3.9 ; P=0.102)$. For the PAC-SYM subscales, the per period abdominal symptom score was lower in the PR naloxone period compared to macrogol $(P=0.002)$ (Fig. 4B). There were no differences in PAC-SYM rectal symptoms score between the treatments $(-0.4 \pm 1.1$ vs $0.0 \pm 1.9 ; P=0.525)$ or constipation symptoms score $(5.0 \pm 3.4$ vs $5.4 \pm 3.8 ; P=0.834)$.

No difference was found for GSRS total score at baseline $(P=$ 0.913), or between treatments on day 5 (PR naloxone vs macrogol) $(P=0.827)$. For the GSRS syndrome dimension scores, abdominal symptoms increased from baseline to day 5 both during PR naloxone $(+38 \%, P<0.001)$ and macrogol $(+21 \%, P=0.020)$, but there was no difference between the treatments on day $5(P=$ 0.171). GSRS constipation score also increased significantly from baseline to day 5 both during PR naloxone $(+6 \%, P<0.001)$ and macrogol $(+16 \%, P<0.001)$, but there was no difference between the treatments on day $5(P=0.933)$. No other differences for GSRS were found (all $P>0.05$ ).

The cumulated per period stool frequency was lower in the PR naloxone period compared to macrogol $(4.2 \pm 1.2$ vs $5.4 \pm 1.5$; $P=0.035$ ). Post hoc analysis revealed a difference on day 4 with a mean number of bowel movements of 0.9 in the PR naloxone period versus 1.9 in the macrogol period $(P<0.001)$, but not on any of the other days (all $P>0.05$ ) (Table 3). The mean Bristol stool
Table 3. Bristol Stool Form Scale Data (Daily Stool Frequency and Stool Type During the 2 Treatment Periods)

\begin{tabular}{lccccc}
\hline \multicolumn{7}{c}{ Day 1 } & Day 2 & Day 3 & Day 4 & Day 5 \\
\hline Frequency & \multicolumn{7}{c}{} \\
PR naloxone & $0.6 \pm 0.4$ & $1.0 \pm 0.4$ & $1.2 \pm 0.5$ & $0.9 \pm 0.3$ & $0.5 \pm 0.2$ \\
Macrogol & $0.5 \pm 0.4$ & $1.1 \pm 0.5$ & $1.3 \pm 0.5$ & $1.9 \pm 0.6$ & $0.6 \pm 0.4$ \\
$P$-value & 0.559 & 0.845 & 0.696 & $<0.001$ & 0.696 \\
Type & & & & & \\
PR naloxone & $3.3 \pm 0.7$ & $3.5 \pm 0.4$ & $3.3 \pm 0.7$ & $3.5 \pm 0.5$ & $2.8 \pm 0.8$ \\
Macrogol & $3.8 \pm 0.4$ & $3.4 \pm 0.5$ & $3.3 \pm 0.5$ & $3.5 \pm 0.5$ & $4.6 \pm 0.6$ \\
$P$-value & 0.999 & 0.999 & 0.999 & 0.999 & 0.001
\end{tabular}

$\mathrm{PR}$, prolonged-release.

Data are presented as means $\pm 95 \%$ confidence interval.

type per period did not differ between PR naloxone and macrogol treatment ( $3.2 \pm 0.4$ vs $3.5 \pm 0.5 ; P=0.141)$. However, pairwise comparison of types between days during the 2 treatments revealed a significant difference on day 5 , but not on any of the other days (all $P>0.05)$ (Table 3).

\section{Associations}

There were no associations between baseline corrected segmental colorectal volumes in any of the colorectal segments and questionnaire scores during either of the treatment periods (all Pearson's $r< \pm 0.5$; all $P>0.05$ )

\section{Discussion}

This study evaluated regional GI transit times, segmental colorectal transit and volume, and GI symptoms during treatment 
with PR oxycodone/naloxone versus PR oxycodone plus macrogol 3350. Colorectal volumes did not change during PR oxycodone/ naloxone treatment, but increased significantly in all segments during PR oxycodone plus macrogol 3350 treatment. However, there were no differences in neither regional GI transit times nor segmental colorectal transit between treatments. Self-assessed GI symptom scores increased during both treatments, but the Patient Assessment of Constipation abdominal symptom score was lower in the PR oxycodone/naloxone period.

\section{Gastrointestinal Transit}

Regional GI and segmental colorectal transit times were similar during the 2 treatment periods and comparable to transit during PR oxycodone alone (44 hours) in a previous experimental study from our group using the same oxycodone dose. ${ }^{11}$ For the PR naloxone period, our results are somewhat in contrast to a scintigraphic transit study by Smith et al, ${ }^{22}$ who showed that a single dose of $10 \mathrm{mg}$ PR naloxone in combination with $20 \mathrm{mg}$ PR oxycodone significantly reduced colonic transit compared to PR oxycodone alone. The reason for this discrepancy between the studies remains obscure, but may reflect the different methodologies used to assess transit times. In our study, measurements rely on transport of a nondigestable solid, whereas Smith et $\mathrm{al}^{22}$ used a radiolabelled resin to measure transit. Moreover, the dosage regimen and the drugs differed between the studies.

Interestingly, macrogol treatment did not decrease transit time in comparison to the study where we administered PR oxycodone alone. ${ }^{11}$ The pathophysiological basis for OIC is caused by hyperpolarization of enteric neurons and reduction in neurotransmitter release thereby inhibiting motility. ${ }^{23}$ Macrogol acts by increasing stool volume through increased hydration, thereby triggering colonic motility. ${ }^{24}$ However, these actions have primarily been studied patients with functional constipation. As macrogol does not target the underlying pathophysiology of OIC, this may explain why there is no significant improvement in transit times.

\section{Segmental Colorectal Volumes}

Change in total colorectal and rectosigmoid volumes were significantly higher after macrogol treatment compared to PR naloxone. This is not surprising, considering that macrogol is an osmotic laxative. ${ }^{24}$ However, coupled with the transit times it is interesting that the increased stool volume during macrogol did not result in a decrease in gastrointestinal transit compared to the PR naloxone period. Moreover, the transit in the macrogol period was similar to our previous study where PR oxycodone was administered alone. ${ }^{11}$ In a study by Lam et al, ${ }^{25}$ the authors used MRI scans to measure intestinal volumes, transit, and motility in response to a single dose of macrogol in patients with either functional constipation or irritable bowel syndrome with constipation. Functional constipation patients showed slower transit and greater ascending colon volumes. Using objective MRI biomarkers, they could distinguish functional constipation from irritable bowel syndrome with constipation with a specificity of $95 \%$. Compared with the current study, it is plausible that OIC shares pathophysiological traits with slow transit constipation, but the volume increase is inadequate to stimulate colonic motility and thus transit.

\section{Questionnaires}

BFI scores increased similarly during both treatments; from 5 to 27 points during PR naloxone treatment and from 6 to 29 points during macrogol. Previous studies have demonstrated that a 12 point increase can be interpreted as clinically meaningful, ${ }^{26}$ but a total score of $\leq 30$ is considered to indicate a normal bowel function. ${ }^{27}$ In our previous study, the BFI score increased 34 points during PR oxycodone treatment. ${ }^{11}$ Thus, both treatments seem to have a minor positive effect on BFI compared to our previous study, although the difference may not be significant. It could also be argued that neither of the treatments was successful in reducing the constipating effects of oxycodone assessed by the BFI scores.

The PAC-SYM questionnaire showed that the abdominal symptom subscale score was lower in the PR naloxone period. This may reflect that the PAC-SYM — as a whole_- evaluates a broader spectrum of more pronounced GI symptoms, as rectal burning, bleeding, or tearing are also part of the questionnaire. ${ }^{21}$ Opioid treatment results not only in constipation, but a range of other symptoms such as reflux, bloating, abdominal distension and cramping, nausea, anorexia and therefore the term OIBD is more appropriate. ${ }^{28,29}$ As the abdominal symptom subscale score covers symptoms as discomfort in the stomach, bloating, and stomach cramps that are consistent with the more broadly description of the pan-intestinal effects of opioids, it appears the participants are better treated with PR naloxone than macrogol. However, these abdominal symptoms may also be ascribed to side effects from the macrogol treatment. ${ }^{30}$ The higher GSRS scores showed a similar pattern with significant increases for abdominal symptoms and constipation, but not for any of the other dimensions.

Stool frequency was lower in the PR naloxone period. Interestingly, PAC-SYM abdominal symptom score was higher in the macrogol period, despite the participants' higher stool frequency, illustrating that stool frequency does not necessarily result in lower symptom scores.

Segmental colorectal volumes and were not associated ques- 
tionnaires scores. This may reflect the challenging clinical picture of constipation where patients' perception of symptoms seldom correspond to objective findings, complicating effective alleviation of symptoms. $^{31}$

\section{Study Limitations and Strengths}

The cancelled MRI scans on day 5 due to 3D-Transit capsule retention-resulting in only 12 participants with full MRI datasets-is a limitation of the study. Despite this, our results clearly indicate that the significant increase in total colorectal and rectosigmoid volumes during macrogol treatment did not improve transit compared to PR naloxone. As Figure 2 also illustrates, the volumes in the ascending, transverse, and descending colon were all numerically higher during macrogol treatment, albeit this was not statistically significant. The missing scans on day 5 significantly reduce the statistical power of the calculations, which may explain the lack of significance in these colorectal segments. In concert, these results suggest that increase in volume do not necessarily result in a decrease in transit time.

Exclusion of women from the study may have introduced gender bias. This was done in order to avoid confounding alterations in gut function related to the menstrual cycle. ${ }^{32}$ However, functional constipation and irritable bowel syndrome are more prevalent in women, suggesting greater susceptibility to alterations in gut function. $^{33,34}$

The experimental design may not mirror the clinical picture of chronic pain patients, who are usually prescribed higher doses of opioids and treated for longer periods. However, previous animal studies suggest that while tolerance may develop to the analgesic effects and upper GI motility upon repeated morphine administration, tolerance does not develop in the colon. ${ }^{35,36}$ To the authors knowledge no similar studies exist in humans, but if the preclinical results can be translated to humans, the outcomes of a clinical study would likely be either similar to ours or potentially more profound due to the longer treatment and/or higher doses. Nonetheless, in clinical studies, confounding factors that can affect gut function, such as concomitant drug use, immobility, dehydration, anxiety, depression, and anorexia are difficult to avoid and may bias results. When these factors are controlled for in an experimental setting, the results may consequently reflect the effect of the treatment of interest to a greater extent.

\section{Conclusion}

Colorectal volumes did not change during PR oxycodone/nal- oxone, but increased significantly in all segments during oxycodone plus macrogol 3350 treatment. Self-assessed GI symptom scores increased during both treatments. However the abdominal symptom score was lower in the PR oxycodone/naloxone period, despite lower stool frequency compared to the oxycodone plus macrogol period. These results indicate that neither oral PR oxycodone/naloxone nor oxycodone plus macrogol are able to reverse opioid-induced delay in GI transit. However, PR oxycodone/naloxone results in a lower abdominal symptom burden than oxycodone plus macrogol consistent with a higher colorectal volume load in the oxycodone plus macrogol period. The objective methods presented may also be useful in future comparative studies treatment of constipation of other causes.

Acknowledgements: We thank Kenneth Krogh Jensen, MRI radiographer, for his assistance with MRI scans and colorectal volume segmentation.

Financial support: This study was funded in part by Innovation Fund Denmark - Individuals, Disease and Society (Grant No. 10092786), in part by The A.P. Møller Foundation for the Advancement of Medical Science (Grant No. 14-319), in part by Aage og Johanne Louis-Hansens Fond, and in part Svend Andersen Fonden, and in part by an unrestricted grant from Mundipharma Research GmbH \& Co. KG.

Conflicts of interest: Asbjørn M Drewes received an unrestricted grant form Mundipharma.

Author contributions: Jakob L Poulsen: study design, data acquisition, data analysis, data interpretation, drafting of manuscript, and statistical analysis; Esben B Mark: data analysis, data interpretation, and drafting of manuscript; Christina Brock: study supervision, study design, data interpretation, and revision of the manuscript for important intellectual content; Jens B Frøkjær and Klaus Krogh: study design, data interpretation, and revision of the manuscript for important intellectual content; and Asbjørn M Drewes: obtained funding for the study, study supervision, study design, data interpretation, and revision of the manuscript for important intellectual content.

\section{References}

1. Argoff CE, Brennan MJ, Camilleri M, et al. Consensus recommendations on initiating prescription therapies for opioid-induced constipation. Pain Med 2015;16:2324-2337. 
2. Lee AA, Hasler WL. Opioids and GI motility-friend or foe? Curr Treat Options Gastroenterol 2016;14:478-494.

3. Gaertner J, Siemens W, Camilleri M, et al. Definitions and outcome measures of clinical trials regarding opioid-induced constipation: a systematic review. J Clin Gastroenterol 2015;49:9-16.

4. Caraceni A, Hanks G, Kaasa S, et al. Use of opioid analgesics in the treatment of cancer pain: evidence-based recommendations from the EAPC. Lancet Oncol 2012;13:e58-e68.

5. Camilleri M, Drossman DA, Becker G, Webster LR, Davies AN, Mawe GM. Emerging treatments in neurogastroenterology: a multidisciplinary working group consensus statement on opioid-induced constipation. Neurogastroenterol Motil 2014;26:1386-1395.

6. Christensen HN, Olsson U, From J, Breivik H. Opioid-induced constipation, use of laxatives, and health-related quality of life. Scand J Pain 2016;11:104-110.

7. Gonenne J, Camilleri M, Ferber I, et al. Effect of alvimopan and codeine on gastrointestinal transit: a randomized controlled study. Clin Gastroenterol Hepatol 2005;3:784-791.

8. Nelson AD, Camilleri M. Opioid-induced constipation: advances and clinical guidance. Ther Adv Chronic Dis 2016;7:121-134.

9. Ford AC, Brenner DM, Schoenfeld PS. Efficacy of pharmacological therapies for the treatment of opioid-induced constipation: systematic review and meta-analysis. Am J Gastroenterol 2013;108:1566-1574.

10. Mearin F, Lacy BE, Chang L, et al. Bowel disorders. Gastroenterology 2016;150:1393-1407, e5.

11. Poulsen JL, Nilsson M, Brock C, Sandberg TH, Krogh K, Drewes AM. The impact of opioid treatment on regional gastrointestinal transit. J Neurogastroenterol Motil 2016;22:282-291.

12. Nilsson M, Poulsen JL, Brock C, et al. Opioid-induced bowel dysfunction in healthy volunteers assessed with questionnaires and MRI. Eur J Gastroenterol Hepatol 2016;28:514-524.

13. Gregersen T, Grønbæk H, Worsøe J, Schlageter V, Laurberg S, Krogh K. Effects of sandostatin LAR on gastrointestinal motility in patients with neuroendocrine tumors. Scand J Gastroenterol 2011;46:895-902.

14. Dipalma JA, Cleveland MV, McGowan J, Herrera JL. A randomized, multicenter, placebo-controlled trial of polyethylene glycol laxative for chronic treatment of chronic constipation. Am J Gastroenterol 2007;102:1436-1441.

15. Worsøe J, Fynne L, Gregersen T, et al. Gastric transit and small intestinal transit time and motility assessed by a magnet tracking system. BMC Gastroenterol 2011;11:145.

16. Hiroz P, Schlageter V, Givel J-C, Kucera P. Colonic movements in healthy subjects as monitored by a magnet tracking system. Neurogastroenterol Motil 2009;21:838-e57.

17. Haase AM, Gregersen T, Schlageter V, et al. Pilot study trialling a new ambulatory method for the clinical assessment of regional gastrointestinal transit using multiple electromagnetic capsules. Neurogastroenterol Motil 2014;26:1783-1791.

18. Fynne L, Worsøe J, Gregersen T, Schlageter V, Laurberg S, Krogh K. Gastrointestinal transit in patients with systemic sclerosis. Scand J Gastroenterol 2011;46:1187-1193.

19. Sandberg TH, Nilsson M, Poulsen JL, et al. A novel semi-automatic segmentation method for volumetric assessment of the colon based on magnetic resonance imaging. Abdom Imaging 2015;40:2232-2241.

20. Nilsson M, Sandberg TH, Poulsen JL, et al. Quantification and variability in colonic volume with a novel magnetic resonance imaging method. Neurogastroenterol Motil 2015;27:1755-1763.

21. Olesen AE, Drewes AM. Validated tools for evaluating opioid-induced bowel dysfunction. Adv Ther 2011;28:279-294.

22. Smith K, Hopp M, Mundin G, et al. Naloxone as part of a prolonged release oxycodone/naloxone combination reduces oxycodone-induced slowing of gastrointestinal transit in healthy volunteers. Expert Opin Investig Drugs 2011;20:427-439.

23. Wood JD, Galligan JJ. Function of opioids in the enteric nervous system. Neurogastroenterol Motil 2004;16(suppl 2):17-28.

24. Cinca R, Chera D, Gruss HJ, Halphen M. Randomised clinical trial: macrogol/PEG 3350+electrolytes versus prucalopride in the treatment of chronic constipation -- a comparison in a controlled environment. Aliment Pharmacol Ther 2013;37:876-886.

25. Lam C, Chaddock G, Marciani L, et al. Colonic response to laxative ingestion as assessed by MRI differs in constipated irritable bowel syndrome compared to functional constipation. Neurogastroenterol Motil 2016;28:861-870.

26. Rentz AM, Yu R, Müller-Lissner S, Leyendecker P. Validation of the bowel function index to detect clinically meaningful changes in opioidinduced constipation. J Med Econ 2009;12: 371-383.

27. Ducrotté $\mathrm{P}$, Caussé $\mathrm{C}$. The bowel function index: a new validated scale for assessing opioid-induced constipation. Curr Med Res Opin 2012;28:457-466.

28. Brock C, Olesen SS, Olesen AE, Frøkjaer JB, Andresen T, Drewes AM. Opioid-induced bowel dysfunction: pathophysiology and management. Drugs 2012;72:1847-1865.

29. Poulsen JL, Brock C, Olesen AE, Nilsson M, Drewes AM. Evolving paradigms in the treatment of opioid-induced bowel dysfunction. Therap Adv Gastroenterol 2015;8:360-372.

30. MedlinePlus. Polyethylene Glycol 3350. Available from URL: https:// medlineplus.gov/druginfo/meds/a603032.html (accessed 12 December, 2017).

31. Johanson JF, Kralstein J. Chronic constipation: a survey of the patient perspective. Aliment Pharmacol Ther 2007;25:599-608.

32. Moore J, Barlow D, Jewell D, Kennedy S. Do gastrointestinal symptoms vary with the menstrual cycle? Br J Obstet Gynaecol 1998;105:13221325.

33. Canavan C, West J, Card T. The epidemiology of irritable bowel syndrome. Clin Epidemiol 2014;6:71-80.

34. McCrea GL, Miaskowski C, Stotts NA, Macrea L, Paul SM, Varma MG. Gender differences in self-reported constipation characteristics, symptoms, and bowel and dietary habits among patients attending a specialty clinic for constipation. Gend Med 2009;6:259-271.

35. Akbarali HI, Inkisar A, Dewey WL. Site and mechanism of morphine tolerance in the gastrointestinal tract. Neurogastroenterol Motil 2014;26:1361-1367.

36. Ross GR, Gabra BH, Dewey WL, Akbarali HI. Morphine tolerance in the mouse ileum and colon. J Pharmacol Exp Ther 2008;327:561-572. 